\title{
Message from the Workshop on the Future of Social Networking
}

\author{
Eiko Yoneki \\ Computer Laboratory \\ University of Cambridge \\ United Kingdom \\ eiko.yoneki@cl.cam.ac.uk \\ Neil Walton \\ Statistical Laboratory \\ University of Cambridge \\ United Kingdom \\ n.s.walton@statslab.cam.ac.uk
}

\author{
Jon Crowcroft \\ Computer Laboratory \\ University of Cambridge \\ United Kingdom \\ jon.crowcroft@cl.cam.ac.uk
}

\author{
Milan Vojnovic \\ Microsoft Research \\ United Kingdom \\ milanv@microsoft.com
}

\author{
Pietro Lio' \\ Computer Laboratory \\ University of Cambridge \\ United Kingdom \\ pietro.lio@cl.cam.ac.uk \\ Roger Whitaker \\ Cardiff University \\ United Kingdom \\ r.m.whitaker@cs.cardiff.ac.uk
}

\begin{abstract}
Electronic social networks are a relatively new pervasive phenomenon that has changed the way in which we communicate and interact. They are now supporting new applications, leading to new trends and posing new challenges. The workshop titled "Future of Social Networking: Experts from Industry and Academia" took place in Cambridge on November 18, 2010 to expose how the future of social networking may develop and be exploited in new technologies and systems. We provide a summary of this event and some observations on the key outcomes.
\end{abstract}

\section{Categories and Subject Descriptors}

C.2.3 [Network Architecture and Design]: Network Operation; I.0 [Computing Methodologies]: General

\section{General Terms}

Design, Economics, Models, Security, Human Factors

\section{Keywords}

Social networks, Privacy, Recommendation

\section{INTRODUCTION}

Electronic social networks are now a key part of everyday life, and frequently used for applications such as communication, debate and maintaining contacts. However, as we become more familiar and dependent on these web-based technologies, social media are increasingly underpinning and being exploited for new applications. The purpose of the workshop, titled the Future of Social Networking: Experts from Industry and Academia was to bring together key people from different sectors to develop a unified view on how the evolution of social networking may occur, how it may be exploited and to identify what challenges we may face. The workshop was held in Cambridge UK on November 18, 2010, in Møller Centre, Churchill College, the University of Cambridge.

The event was timely and preceded major political events in which social media were important. What role did social media play in recent political movements in Tunisia or Egypt? Did Twitter or Facebook take on an important task in these revolutions? Before the Internet and mobile phones were shut off, Twitter, Google and
Facebook were used to spread the word for organising demonstrations among protesters, and social media possibly made it spread faster. The role of social media is expanding, and everybody will eventually be part of building and contributing contents to social media.

What would be the task of academia in these emerging social phenomena? How would industry offer products and tools that interface to the users directly? How do we bridge and split the task in this emerging research topic between academia and industry? In order to address such questions, we have organised this workshop.

The workshop was organised as part of the EU FP7 SocialNets project, Social networking for pervasive adaptation - Harnessing adaptive human social structures for tomorrow's wireless networks [3]. See the workshop web page http://www.cl.cam.ac.uk/research $/ s r g / n e t o s / s o c i a l n e t s$ for further details of the project. The project is a part of the Pervasive Adaptation Initiative (PERADA) started in the FET section of the 7th Framework Programme within Objective ICT-2007.8.2 of the 'Future Emerging Technologies'. With many other researchers, we have been studying fundamental aspects of these new systems that now pervade our lives, looking at the underpinning theories that explain the structure and dynamics of the networks from a mathematical physics perspective and from the anthropological viewpoint.

We are interested in the consequences for this understanding in terms of systems that we and others build in practice for experiment or for fun and profit. The objectives of the workshop were twofold: (1) to see what outputs from our research might be useful to practitioners and (2) to see what problems industrials (and their customers in society) were encountering that will form inputs to interesting challenges for researchers to tackle most fruitfully. Especially, our focus is on adaptivity for communication in emerging technology-rich environments. We aim at providing the traditional notion of network-based communication by structuring opportunistic networking with new levels of functionality and agility, where social networks are exploited.

The organisers were mainly the members of the project from the University of Cambridge Computer Laboratory together with Microsoft Research in Cambridge and the statistical laboratory in the University of Cambridge. In Microsoft Research in Cambridge, the research focus on social networks includes algorithmic aspects, design of systems and services, and understanding of user behaviour, e.g. [5]. 
The workshop came out of a perceived need to match make between industry and research needs for future work in the context of Online Social Networks. The main goal of the workshop was the focus on the commercial exploitation of social networks. We gathered contributions from practitioners in industry, as well as academic and industrial researchers, to discuss new ideas on the theme of social networking and computer systems. Online social networks are among the most popular sites on the Web and continue to grow rapidly. They provide a means for establishing identities, sharing information, and creating relationships, while the resulting social graph can provide a basis for communicating, distributing, and locating content. The workshop consisted of 5 featured speakers and 7 regular speakers including poster/demo session with 12 posters and an open panel discussion. Speakers spanned several major industry research laboratories, start-ups, and academic institutions. The five Featured Speakers were:

- Richard Allan (Facebook, UK)

- Christos Gkantsidis (Microsoft Research Cambridge, UK)

- Sharad Goel (Yahoo! Research, USA)

- Balachander Krishnamurthy (AT\&T Research, USA)

- Jurgen Van Gael (Microsoft FUSE Labs, UK)

The number of registrations to the workshop was 115, and the actual total number of attendees was around 100 on the day. Some people came or left in the afternoon. On average, about 80 people participated in the entire workshop. The workshop was lively for the whole day both during the talks, buffet lunch time and the breakout times. The participants came from universities, industrial research institutes, and local companies developing products related to social networking, which made the workshop an interesting melting pot for those working in social networks related domains.

The workshop blog site, Wiki page, and Twitter hashtag (\#socialnetsws) were set up for real time online social communication for the workshop. We planed to explore such social media as a tool to accelerate the workshop discussion. However, the Internet facility at the venue was not good enough to support the Internet connection to all the participants and it did not reach the success as we originally expected in this attempt. The number of tweets was about 150 and blog entries recorded 15 . In this occasion, Wiki did not get used at all.

During the workshop, the following experiment was conducted. The purpose of the experiment was to explore the correlation between actual viral message propagation and physical proximity.

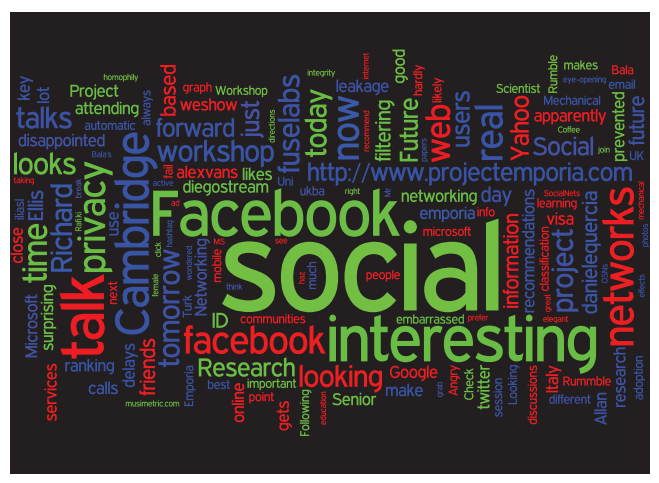

Figure 1: Wordle Output of Keywords in tweets

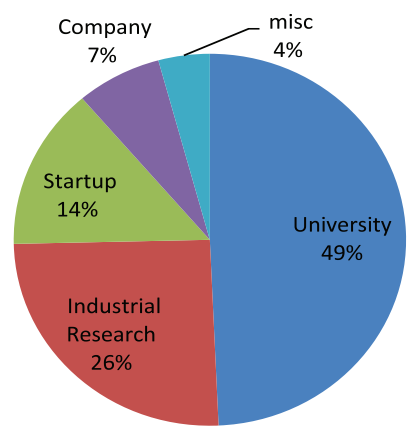

(a)Participant Affiliation w/o Univ. Cambridge

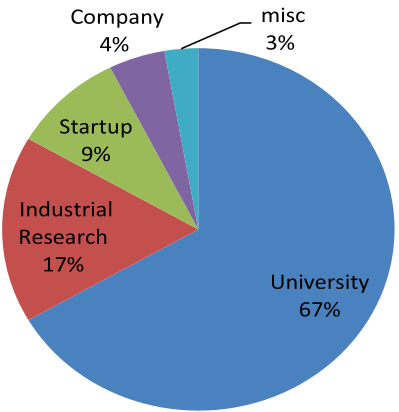

(a)Participant Affiliation with Univ. Cambridge
Figure 2: Affiliation of Participants

The result can possibly be correlated to the social graph of the online social networks.

- Location tracking of participants' mobile phones: We placed 20 Intel motes (sensor board) at the landmarks of the conference venue. Those sensors detected the mobile phones, which sets Bluetooth discoverable.

- Viral rumour propagation: We started a rumour 'Google is opening a new research lab in Cambridge' from 5 people. At the end of the workshop, we planned to obtain the number of people who heard the rumour.

Figure 1 displays the Wordle [10] output produced by all keywords in the tweets gathered by using the hashtag. This highlights the dominant keywords such as 'privacy' and 'facebook'.

Figure 2(a) shows the affiliation of participants without ones from the University of Cambridge and Figure 2(b) depicts it with the University of Cambridge. Without the participants from the University of Cambridge, the participation from academia and industry was roughly balanced.

\section{TALKS}

The workshop program consisted of 5 featured talks each of duration of 45 minutes and 7 regular talks each of duration of 15 minutes. In 5 featured talks, 4 of speakers were from the industrial research laboratories and 1 from Facebook. In our original program we also had as a featured speaker Vahab S. Mirrokni, from Google in New York, but due to issues with getting a visa for entry into UK in time, his talk was canceled.

All the talks brought interesting questions and discussions. Key issues raised through debate concerned the issue of privacy and how this is no longer a 'hard' constraint in the design and operation of systems. Effectively we are willing to trade privacy for functionality, and private data is a commodity of great commercial value. It was noted that these issues are going to get more important as social networks increasingly have a geo-spatial component, which informs about our mobility and physical behaviour.

The program including the abstract of the talk, slides, and video can be found in [9]. A brief summary of talks can be found below.

\subsection{Featured Talks}

The first talk was by Sharad Goel from Yahoo! Research with the title 'Birds of a Feather Shop Together: Predicting Adoption with Social Networks'. His message was 'what your friends do, there's more than $50 \%$ chance you will' and 'if your friends click on 
an ad, you more likely to click on an ad'. Thus adoption is predicted using individual-level attributes such as age, sex, and geographic location. The principle of homophily suggests that social data (e.g., the attributes of people with whom one is in contact) might also have predictive value.

an experimental web service that sifts through news streams in real time to bring stories of interest to users, categorized by topic area and ranked for relevance.

Jurgen Van Gael introduced the Project Emporia of Microsoft FUSE Lab in a talk with the title 'Making Sense of the Information in the Real Time Web'. Emporia is a web service that provides personalised information to users by using real time filtering and recommendation, which can be seen as an automated Digg [1]. The service uses as input the information collected from various sources such as Twitter and other social media to feed a recommendation engine. The talk addressed the interaction of automatic classifiers on tweets, human-based classification using Amazon's Mechanical Turk [4], and the underlying collaborative filtering engine.

Richard Allan, Facebook's Director of Policy in Europe, gave a talk 'Industry Directions: Where Social Network Providers are heading'. Richard described recent developments of the Facebook service and outlined future trends for where it is heading. The Facebook is integrating other services such as location services, mobile applications, search, and others. For example, integration with gaming is an emerging area and the balance between gaming and social system is shifting. The Facebook has a 'site integrity team' ensuring users use real identification. However, Middle Eastern users have male and female accounts for different circles to deal with their religious background.

Balachander Krishnamurthy from AT\&T Research gave a powerful talk 'Privacy Leakage on Online Social Networks'. Interestingly this talk highlighted the potential risks we are facing from combining our identity and online activity. It was an eye-opening talk; your ID is potentially leaked whilst using social networks. All your information belongs to the social network provider. At the end how much you want to preserve privacy depends on a business model for the sharing of your private personal information.

Christos Gkantsidis from Microsoft Research Cambridge presented a talk with the title 'Hermes: Clustering Users in LargeScale E-mail Services'. The main idea was to design new and efficient systems by leveraging the information about user interactions. Specifically, the system leveraged the information about email information flows among enterprise users in order to optimise assignment of email data across geographically dislocated exchange servers. This is an example where the information about an online social graph can be used for system design.

\subsection{Short Talks}

There were 7 short talks and 4 of them are from small companies producing social network based products. The rest of short talks were from academics from universities.

Richard Millington from FeverBee talked about 'The Online Community Development Process'. Richard addressed 3 questions: 1) what is the optimal group size for maximum efficiency? 2) how does influence spread amongst a group? and 3) how do variations in culture impact the community development process? He emphasised the importance of actual process of building a meaningful online community from the very beginning.

Andrea LaRocca from Rafi.ki introduced 'The Future of International Education: Rafi.ki, a Network of Learners', where the social network is used for education. The principle is that students want to learn from their peers! Andrea's talk was refreshing since she presented real evidence of using online social networks in the domain of education.

Andrew J Scott from Rummble presented 'The Best Advice Does Not Always Come From Your Friends' and showed his company's products such as providing mobile personalised spatial recommendations. He made some interesting assumptions such that the market will fix privacy. The best recommenders are those who share the same tastes and financial status, which indicates the same statement of Sharad's point.

Mischa Tuffield from Garlik Ltd gave a talk 'Finding and Future Direction of the W3C Social Web Incubator Group (SWXG)'. He discussed future W3C efforts in and around the Social Web, where W3C pushes for interoperability between different social networks.

Joseph Bonneau from the University of Cambridge, Computer Laboratory Security Group talked about 'Authentication in the Social Web'. Websites increasingly allow users to share all or some of their identity across websites and he addressed new demands for authentication and identity management online and discussed the complicated state of authentication on the web.

Teresa Domenech from UCL gave a talk 'Industrial Symbiosis: Networking for Improved Environmental Performance'. Industrial symbiosis (IS) explores the inter-company dimension in moving towards more closed-loop industrial systems by looking at networks of industrial companies exchanging waste flows and sharing resources. She discussed the networking dynamics of IS networks and their contribution to innovation and to the triple bottom line of organisations.

Richard Mortier from the Nottingham University talked about 'Horizon-Becoming Dataware'. He discussed many different challenges surrounding collection and exploitation of personal digital contextual footprints. Building an ecosystem around digital footprints that maintains acceptable levels of privacy for individual level as part of group is key to enabling growth in value of our social and personal data.

\section{POSTERS}

The poster session provided a forum for researchers and industry to showcase new and emerging work and obtain feedback from workshop attendees. The topics of posters were ranging from crowdsourcing, privacy concern on routing, location sharing, mobile social networking, to community evolution. There were 12 posters in total, which overall the participants evaluated to be of high quality. See [6] for the abstracts of posters.

\section{POST WORKSHOP SURVEY}

A week after the workshop, we conducted a survey with the following nine questions. Among about 100 participants, we received 31 survey answers. A summary is given below.

1. Which session did you attend?

2. Describe 3 keywords that remained in your head after the workshop.

3. Pick 3 of you favorite talks.

4. Pick 3 of your favorite posters.

5. How was the quality of the Internet access?

6. Did you use Twitter/Blog/Wiki?

7. Were you aware of the rumour experiment?

8. Describe your comments on the workshop.

9. Any comment on poster session, open discussion, lunch, coffee area. 


\begin{tabular}{|c|c|c|c|c|}
\hline Keyword & Occurrence & Security & Adv/Rec & Network \\
\hline Privacy & 18 & $\checkmark$ & & \\
\hline Community & 6 & & & $\checkmark$ \\
\hline Social graph & 6 & & & $\checkmark$ \\
\hline Recommendation & 5 & & $\checkmark$ & \\
\hline Security & 4 & $\checkmark$ & & \\
\hline Facebook & 3 & & & \\
\hline Network & 2 & & & $\checkmark$ \\
\hline rafi.ki & 2 & & & \\
\hline Social & 2 & & & \\
\hline Emporia & 2 & & & \\
\hline Academia & 1 & & & \\
\hline Adoption & 1 & & & \\
\hline Advertising & 1 & & $\checkmark$ & \\
\hline Aggregation & 1 & & & \\
\hline Causation & 1 & & & \\
\hline Clustering & 1 & & & \\
\hline Data & 1 & & & \\
\hline Enthusiasm & 1 & & & \\
\hline Friends & 1 & & & $\checkmark$ \\
\hline Geosocial & 1 & & & \\
\hline Good people & 1 & & & \\
\hline Google & 1 & & & \\
\hline Hermes & 1 & & & \\
\hline Interdisciplinary & 1 & & & \\
\hline Leakage & 1 & $\checkmark$ & & \\
\hline Market forces & 1 & & $\checkmark$ & \\
\hline Matchbox & 1 & & & \\
\hline Mobile & 1 & & & \\
\hline Online Social Network & 1 & & & $\checkmark$ \\
\hline Opportunities & 1 & & & \\
\hline Random & 1 & & & \\
\hline Rumble & 1 & & $\checkmark$ & \\
\hline Rumour & 1 & & & $\checkmark$ \\
\hline Smart people & 1 & & & \\
\hline Social influence in marketing & 1 & & $\checkmark$ & \\
\hline Structure & 1 & & & $\checkmark$ \\
\hline Web architecture & 1 & & & \\
\hline Yahoo & 1 & & & \\
\hline
\end{tabular}

Table 1: Keywords that remained in participants' heads

Most of participants attended in the morning sessions and the first afternoon session until 15:00, however 80\% of participants stayed until the end of workshop. Further detail of the survey result can be found in [2].

\subsection{Memorable Keywords}

Table 1 shows the list of keywords that participants indicated as memorable after the workshop. The three columns (i.e. 3rd, 4th and 5th) depict the classification of the keywords to the related topics. Overall, 'Privacy' and 'Security' dominate, followed by 'Recommendation' and 'Community'. While this could have been influenced by the format of the talks, it indicates possible areas of main concern when people deal with social networks and suggest directions for future research.

Figure 4 shows the top 10 popular keywords from the first choice, while Figure 5 depicts the top 10 from all keywords listed by the

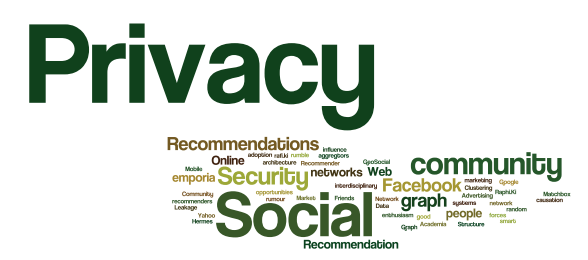

Figure 3: Wordle Output from Memorable Keywords participants. The word 'privacy' was significant in both rankings.

Figure 3 displays the Wordle output produced by all memorable keywords. This highlights the dominant keyword 'Privacy'.

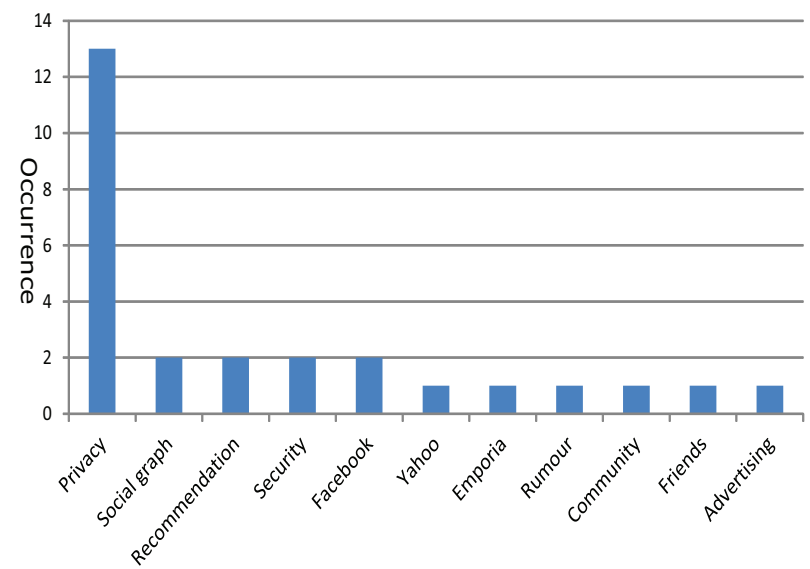

Figure 4: Keyword Popularity: Top 10 from First Choice Keywords

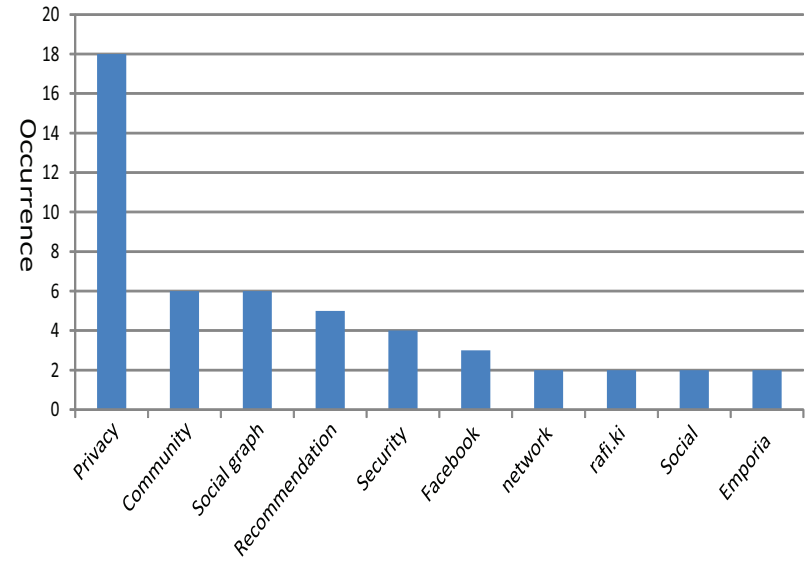

Figure 5: Keyword Popularity: Top 10 from All Keywords

\subsection{Popular Topics in the Talks}

The participants have listed their 3 top favourite talks in the survey among the talks listed in Table 2 (see also [8][9] for the abstract, slides and video of talks).

Balachander Krishnamurthy's talk 'Privacy Leakage on Online Social Networks' is the most popular talk followed by three talks that addressed recommendation systems or information filtering systems in relation to social networks. The result of the survey highlights the audience's two strong topics of interest: 'Privacy' and 'Recommendation'.

\subsection{Popular Posters}

The poster session have covered a good variety of topics ranging from privacy to crowdsourcing, where people's favorites are more spread out. Each poster's abstract can be found in [6]. The participants have listed their top 3 posters in the survey. The most popular 


\begin{tabular}{|l|l|}
\hline Title & Speaker \\
\hline T1. Birds of a Feather Shop Together & Sharad Goel (Yahoo! Research, USA) \\
T2. Making Sense of the Information in the Real Time Web & Jurgen Van Gael (Microsoft FUSE Labs, UK) \\
T3. The Online Community Development Process & Richard Millington (FeverBee) \\
T4. Industry Directions - where social network providers are heading & Richard Allan (Facebook, UK) \\
T5. The Future of International Education: Rafi.ki, a Network of Learners & Andrea LaRocca (Rafi.ki) \\
T6. The Best Advice Does Not Always Come From Your Friends & Andrew J Scott (Rummble) \\
T7. Privacy Leakage on OSNs & Balachander Krishnamurthy (AT\&T Research, USA) \\
T8. Hermes: Clustering Users in Large-Scale E-mail Services & Christos Gkantsidis (Microsoft Research,UK) \\
T9. Finding and Future Direction of the W3C Social Web Incubator Group (SWXG) & Mischa Tuffield (Garlik Ltd) \\
T10. Authentication in the Social Web & Joseph Bonneau (Univ. Cambridge) \\
T11. Industrial Symbiosis: Networking for Improved Environmental Performance & Teresa Domenech (UCL) \\
T12. Horizon- Becoming Dataware & Richard Mortier (Nottingham University) \\
\hline
\end{tabular}

Table 2: Titles of Talks

poster was 'Analysis of content and activity in geo-social networks for place recommendations' and second place was shared among 3 posters (See Figure 6). The topics 'Recommendation' and 'Privacy' also exhibit strong interests among the audience in the poster session (see [7] for the title of posters).

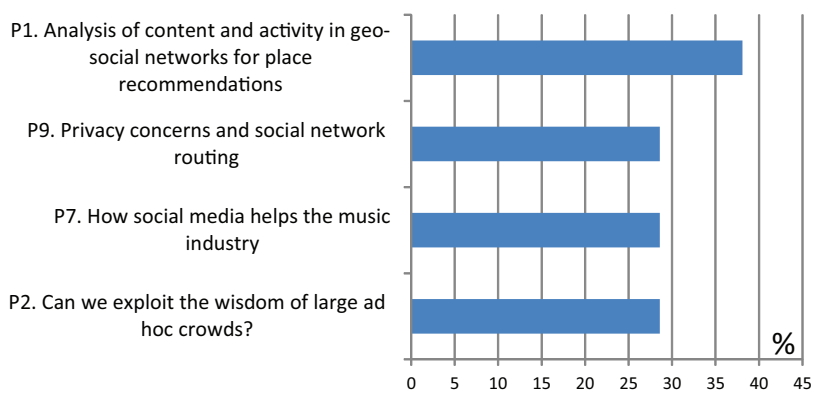

Figure 6: Top 4 Popular Posters

\subsection{Twitter/Blog/Wiki}

The survey shows that only $30 \%$ of participants (among survey takers) were able to access the Internet intermittently. Wiki was not useful in the workshop at all. The shared blog did not work, either. On the other hand, Twitter looks like a good communication means in workshop settings. It is pity that the quality of the Internet access was low.

\section{CONCLUSIONS}

The workshop was of great benefit in clarifying the significant role of academic research for the development of social networking. The workshop also gave clear indications on how social networking may be exploited in future. It was also interesting to see that social networks are exposing many human behaviours and psychological traits that have been previously difficult to deduce. Understanding both physical and social behaviours and exploiting these has been a very important aspect of the SOCIALNETS project, but the workshop has reinforced that academia is very much at the beginning of understanding and exploiting social computing. The outcome from this workshop is very useful for the project, for building up a strong community in this area from both academia and industry.
At the end of the workshop, it was clear that there is an emergent discipline in the area of online social networks, which consists of an intersecting of network science, social and anthropological science, and communications systems. We plan to run a similar workshop in the future to keep community members together and to evolve the research agenda.

At the same time, one possible practical outcome of the workshop may be the creation of a top-tier conference as a target for the work carried out by this community. There is clearly enough focus and a real discipline, however, we are still seeking feedback on whether there is sufficient size to warrant a full conference, or whether the existing venues cater well enough (in a less focused way). The workshop organisers would welcome the readers' input on this.

\section{Acknowledgment}

The research is part funded by the EU grants for the Socialnets, 217141.

\section{REFERENCES}

[1] Digg: Social News Website. http://digg.com/.

[2] EU FP7 SOCIALNETS Deliverable D5.4: Workshop, Wireless Market Report and Exploitation Assessment . http://www.social-nets.eu/deliverables.html.

[3] EU FP7 SOCIALNETS: Social Networking for Pervasive Adaptation. http://www.social-nets.eu/.

[4] Mechanical Turk - Amazon Mechanical Turk. https://www.mturk.com/mturk/welcome.

[5] Microsotf Research Cambridge: Social Networking Research Area. http://research.microsoft.com/en-us/projects/snra/.

[6] Socialnets Workshop: Poster Abstract. http://www.cl.cam.ac.uk/research/srg/netos/socialnets /posters/posters_abstracts.pdf.

[7] Socialnets Workshop: Poster List. http://www.cl.cam.ac.uk/research/srg/netos /socialnets/program.html\#posters.

[8] Socialnets Workshop: Program. http://www.cl.cam.ac.uk/research/srg/netos /socialnets/program.html.

[9] Socialnets Workshop: Talk Abstract. http://www.cl.cam.ac.uk/research/srg/netos/socialnets /talks.pdf.

[10] Wordle. http://www.wordle.net/. 